Old Dominion University

ODU Digital Commons

VMASC Publications

Virginia Modeling, Analysis \& Simulation Center

2016

\title{
Panic That Spreads Sociobehavioral Contagion in Pedestrian Evacuations
}

Terra Elzie

Old Dominion University

Erika Frydenlund

Old Dominion University, efrydenl@odu.edu

Andrew J. Collins

Old Dominion University, acollins@odu.edu

R. Michael Robinson

Old Dominion University, rmrobins@odu.edu

Follow this and additional works at: https://digitalcommons.odu.edu/vmasc_pubs

Part of the Social Psychology Commons, Transportation Commons, and the Transportation Engineering Commons

\section{Repository Citation}

Elzie, Terra; Frydenlund, Erika; Collins, Andrew J.; and Robinson, R. Michael, "Panic That Spreads Sociobehavioral Contagion in Pedestrian Evacuations" (2016). VMASC Publications. 13.

https://digitalcommons.odu.edu/vmasc_pubs/13

\section{Original Publication Citation}

Elzie, T., Frydenlund, E., Collins, A. J., \& Robinson, R. M. (2016). Panic that spreads sociobehavioral contagion in pedestrian evacuations. Transportation Research Record: Journal of the Transportation Research Board, 2586, 1-8. doi:10.3141/2586-01

This Article is brought to you for free and open access by the Virginia Modeling, Analysis \& Simulation Center at ODU Digital Commons. It has been accepted for inclusion in VMASC Publications by an authorized administrator of ODU Digital Commons. For more information, please contact digitalcommons@odu.edu. 


\title{
Panic That Spreads Sociobehavioral Contagion in Pedestrian Evacuations
}

\author{
Terra Elzie, Erika Frydenlund, Andrew J. Collins, and R. Michael Robinson
}

Crowds are a part of everyday public life, from stadiums and arenas to school hallways. Occasionally, pushing within the crowd spontaneously escalates to crushing behavior, resulting in injuries and even death. The rarity and unpredictability of these incidents provides few options to collect data for research on the prediction and prevention of hazardous emergent behaviors in crowds. This study takes a close look at the way states of agitation, such as panic, can spread through crowds. Group composition-mainly family groups composed of members with differing mobility levels-plays an important role in the spread of agitation through the crowd, ultimately affecting the exit density and evacuation clearance time of a simulated venue. This study used an agent-based model of pedestrian movement during the egress of a hypothetical room and adopted an emotional, cognitive, and social framework to explore the transference and dissipation of agitation through a crowd. The preliminary results reveal that average group size in a crowd is a primary contributor to the exit density and evacuation clearance time. The study provides the groundwork on which to build more elaborate models that incorporate sociobehavioral aspects to simulate human movement during panic situations and account for the potential for dangerous behavior to emerge in crowds.

Pedestrian evacuation and crowd movement have obvious implications for transportation, venue, and emergency managers. However, pedestrian modeling and research often neglect to account for group dynamics in the crowd, instead treating independent, individual human movement as analogous to that of gas particles or fluids (1-4). Reduced to physics equations, much of the sociobehavioral decision making that influences individual members of groups within a crowd is lost and leads to oversimplification of the evacuation scenario. Attempting to fill this gap, contemporary pedestrian research and modeling have focused on agent-based or cellular automata modeling techniques that incorporate individual decision making within a group context $(1,5-8)$.

This study built on previous agent-based and cellular automata models to understand how groups might manifest panic-like behaviors in a way that dissipates or intensifies through a crowd. The model allows for individual and group-level behaviors as agents egress from a building through a centralized exit. The model incorporates

T. Elzie, Modeling, Simulation, and Visualization Engineering Department, Old Dominion University, 1300 Engineering and Computational Sciences Building, Norfolk, VA 23529. E. Frydenlund and R. M. Robinson, Center for Innovative Transportation Solutions, Old Dominion University, 1030 University Boulevard, Suffolk, VA 23435. A.J. Collins, Virginia Modeling, Analysis, and Simulation Center, Old Dominion University, 1030 University Boulevard, Suffolk, VA 23435. Corresponding author: T. Elzie, tpett003@odu.edu.

Transportation Research Record: Journal of the Transportation Research Board No. 2586, Transportation Research Board, Washington, D.C., 2016, pp. 1-8. DOI: 10.3141/2586-01 a recently developed emotional, cognitive, and sociobehavioral framework, called Agent_Zero, for modeling the spread of ideas or emotions through populations (9). Although the model is still in its preliminary development stage, it has established the groundwork for future studies of panic and crushing behaviors that occasionally emerge in crowded pedestrian environments. This study has direct relevance for transportation, venue, and emergency planners as they look to simulation models to understand potential egress scenarios.

This paper first provides an overview of pedestrian modeling research relevant to the study of group behavior. A brief description of the Agent_Zero framework follows before moving on to describe the model implementation. Closing the paper is a discussion of the preliminary modeling results and the future development path for this pedestrian model.

\section{PEDESTRIAN EVACUATION AND AGENT_ZERO OVERVIEW}

\section{Pedestrian Evacuation}

The study of pedestrians in egress scenarios constitutes a complex body of research that involves sociological, psychological, and engineering considerations that manifest dynamically through individualand group-level decision making. Inspired by principles from physics, Helbing and Molnar paved the way for modeling human movement in crowds by introducing the social forces model (2). Matarić added to the development of human crowd movement models by identifying basis behaviors - namely, avoidance, safe wandering, following, aggregation, dispersion, and homing - to describe pedestrian behaviors (10). Goal-seeking behaviors and individual movement through crowds laid the foundational work for adding realism to models and simulations of pedestrian scenarios (11-14).

Most real-world pedestrian contexts do not involve entire populations of individuals operating independently, so one facet of the research in this area has examined group behavior and formation to reflect crowd composition more accurately $(7,8,15-18)$. Some pedestrian research has examined the emergence and composition of group structures through individual behaviors $(19,20)$. The study reported here took the existence of groups as a given and pursued greater understanding of pedestrian egress in an environment where groups of varying sizes interact during a venue evacuation $(6,21)$.

Prior research has shown that the presence of groups makes a difference in evacuation time $(6,22)$. Pedestrian movement in models and simulations encompasses a range of factors related to groups, from individual mobility to behaviors adjusted to accommodate group configurations. Most often, individuals maintain a desired speed that is influenced by their own mobility limitations (for example, physical disability, reliance on mobility devices, or age) as well as their 
response to group movement and dynamics $(12,15,18,23)$. Altruism also features in pedestrian models, as group members help one another during egress $(15,18)$. Some influences of altruism are reflected in the current model, as groups adjust speeds to accommodate and assist slower members. Particularly relevant to this model is the idea of group cohesion; that is, certain forces keep group members together as they evacuate toward an exit (5).

In a crowd, an individual's actions are the result of an accumulation of factors, such as past experiences, the emergency level, or observance of others' behaviors. Events occurring in the immediate environment also have a strong influence $(9,24)$. Past studies have used various approaches to capture the human decision making that drives behavior during an evacuation. For example, the Belief-Desire-Intention (BDI) framework (25) is one of the most popular among researchers modeling human decision making during evacuation scenarios (26-28). A BDI agent is characterized by his or her mental state, with three major components: beliefs, desires, and intentions. According to Shendarkar and Vasudevan (26, p. 546),

[b]eliefs correspond to information the agent has about the world. It may be incomplete or incorrect. Desires represent the state of affairs that the agent would wish to be brought about. Intentions represent desires that the agent has committed to achieve.

For instance, in an emergency evacuation simulation, an agent's desire is to leave the current hazardous location as quickly as possible using the fastest and safest path while following beliefs and taking the necessary actions based on intentions (29). Evacuation studies using BDI agents add social complexity to the model by extending the framework to include human relations $(27,28)$.

\section{Agent_Zero Overview}

The model presented here implements a framework similar to BDI to support a better understanding of interpersonal influences in groups and crowds. Agent_Zero, a framework developed by Epstein (9), is an attempt to quantify the behavior leading to an individual acting in a way that influences the behavior of others. The framework provides an explicit computational mechanism with which to understand how, with very little initial intent, certain behaviors emerge from crowds. This wave of behavioral change ripples through the system in unexpected ways and leads to emergent crowd properties. In pedestrian crowds, the framework could be thought of as the natural pushing between individuals leaving a stadium after an event. In most cases, the pushes are normal and tolerated. Occasionally, this pushing precipitates crushing behavior with potentially fatal consequences (30-32).

Epstein expressed his curiosity about these types of phenomena and the motivation to develop Agent_Zero in the following statement (9, pp. 2-3):

People often do things in groups that we would not do alone... We do things for which we have no basis in evidence ... we do them knowing we have no evidence, and sometimes, despite this, we are even the first in the group to do them. [Therefore, the premise is not] to characterize the rational behavior, but to generate behavior that is far from rational.

The Agent_Zero framework is based on the premise that individual emotional, cognitive, and social components influenced by interactions with others drive human behavior (9). The power of this approach comes from its generality, which allows its application to a variety of disciplines, including sociology, psychology, and national defense. The framework enables the exploration of how panic can arise and spread in an otherwise calmly evacuating crowd.

In some cases, even when an individual has no compulsion to act a certain way, that individual may become the one who acts first and subsequently shapes group action. The framework driven by emotional, cognitive, and social factors allows for investigation of how certain behaviors - such as increased aggression, decreased patience, pushing, or running - might emerge from very simple individual choices and influence the outcome of the evacuation clearance time. Agent_Zero provides the mechanism with which to trigger the start of an increasingly agitated crowd.

\section{EVACUATION OF A PEDESTRIAN VENUE}

The pedestrian evacuation model described here was developed with the NetLogo platform for agent-based modeling (33). The simulation environment represents a room with one centralized exit (Figure 1). The environment is purposefully simplistic - that is, free of obstacles, corridors, counterflow, or choices between exits- to allow full exploration of the emotional, cognitive, and social influences on evacuation behavior and decision making. Elimination of the complicated agent heuristics for navigating a more complicated environment eliminates some of the interacting factors that might complicate analyses of whether a social or emotional contagion-type model can provide useful insight into the way pedestrians evacuate a venue. Future versions of this model will involve more complicated environments and decision-making features of pedestrian navigation.

At the start of the simulation, agents are randomly dispersed throughout the environment. During the simulation, individual and grouped agents navigate to avoid collisions with each other while moving toward the exit. Speeds and group cohesion strengths are randomly assigned to individuals, making agents heterogeneous on a number of factors. The model modifies the Agent_Zero components to simulate pedestrians' behaviors and their propensities to become more agitated when evacuating a building. Furthermore, embedded in the mathematical equations quantifying emotional, cognitive, and social factor updating mechanisms, calming agents are introduced to represent authoritative figures, such as peace officers or venue staff whose roles are to de-escalate crowd-level agitation.

\section{Group Dynamics and Exiting Behaviors}

At the core of the pedestrian agent-based model, autonomous, heterogeneous agents maneuver through a crowded environment toward a centralized exit. Many of the assumptions adopted to develop these agents' behaviors derive from earlier qualitative research based on surveys of reported behavior during egress with less mobile group members. The agents comprise groups of varying sizes, from one to 15 people, and attempt to maintain group configurations during the simulated egress.

Agents have two primary goals during the simulation: $(a)$ to stay with predefined group members (cohesion) and (b) to exit the venue. Since agents have heterogeneous walking speeds, the first goal requires that the members of each group adjust their speeds to accommodate less mobile members. The slowest member has a weighted influence on the overall group speed, allowing groups to distribute the burden of slower members and thereby increase the overall group egress rate. This reflects the idea that slower members in the real world, 


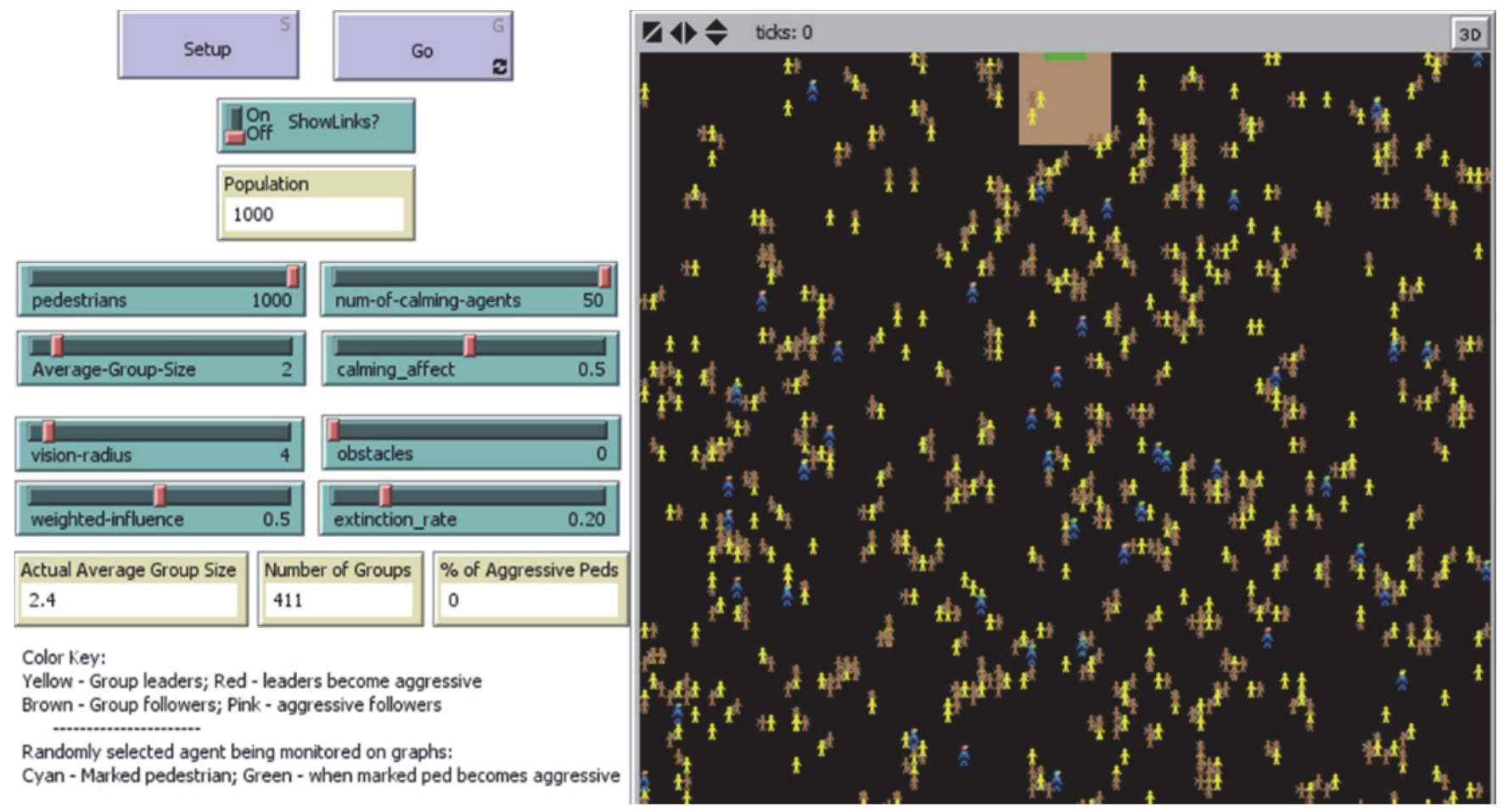

FIGURE 1 Model interface and simulation environment (peds $=$ pedestrians).

such as the elderly or children, could be helped along by other members in the group. Although this help might increase the speed of the slowest members, it still amounts to some decrease in overall group speed. Equation 1 shows the weighted group speed based on the slowest member:

GroupSpeed $=(1-\delta) S_{G}+\delta \cdot s$

where

$\delta=$ weight of the slowest member's speed,

$S_{G}=$ unweighted group speed, and

$s=$ speed of the slowest member.

Here, if $\delta=1$, the slowest member dictates the entire group's speed; if $\delta=0$, the group distributes the entire burden of the slowest member and helps the whole group move faster. This accounts for actions such as carrying small children through a dense crowd. In the current version of the model, groups cannot split apart; thus, the slowest member always has some influence on the overall speed of the group, as other members wait for the slowest to catch up.

Another contribution to the group configuration is that agents in the model have varying preferences for the distance they walk from their predefined groups. Some agents are willing to walk farther from their groups, say, to navigate in a different direction around an obstacle and meet up with the rest of the group on the other side. Others prefer to have very close contact with the members of the group and will not stray far as the group moves toward the exit. This feature attempts to replicate the diversity of group structures in which certain individuals - for example, teenagers — might embody loose bonds of group cohesion, whereas families with small children might prefer to walk closer together (34). Although some pedestrian models focus on the group space (5), the groups in this model have a predetermined leader agent chosen for having the fastest preferred walking speed. Implied in this framework is the intent to emphasize the representation of groups with generally well-defined leaders, such as families with dependents.

The current implementation of this model envisions groups to be family-like structures. Thus, the group is centered on an individual rather than an average central location in the group. The heterogeneity of individual preferences allows some agents to stray farther from the group, but the leader will take the initiative to ensure that all group members are within his or her preference for group distance while navigating toward the exit. Future versions of the model may consider alternative arrangements that would be representative of less hierarchical group structures, such as peers or even acquaintances. In line with this assumption of family group structures, groups in the model maintain their composition throughout the egress period. Group members will wait for slower members before proceeding to the exit.

In addition, the main contributing factors in the model for the agents' decision-making processes are

- Emotional: conditioned learning through associations;

- Cognitive: reasoning based on the agitation of others;

- Social: overall disposition based on the disposition of others; and

- Action rule: act or not act on a behavior.

The following section discusses each of these factors and their impacts on agent behavior in the model.

\section{Behavior Dispersion Through a Crowd}

For each defined group, the model randomly selects one agent to become the group leader while the others in the group become the 
followers. This assumption simplifies the Agent_Zero implementation, as only the group leader is endowed with relevant cognitive, emotional, and social decision-making properties. Although only the leader adjusts his or her level of agitation, members of the group adjust their behavior to reflect that of the leader. In this way, groups manifest the same levels of aggression across all members, as instigated by their leaders.

Elevated levels of agitation in this model cause the agents to increase their speed, simulating pedestrians beginning to run toward an exit. As the pedestrians navigate to the exit, the calming agents gravitate toward those agents that display the most agitation. The presence of calming agents de-escalates individual agitation levels by some small amount $(35,36)$. The counteracting interactions of agents escalating one another while calming agents attempt to decrease crowd-level agitation (24) create nonlinear emergent effects in the model that arise from individual interactions but affect group-level evacuation clearance times and overall agitation levels. These dynamics begin to reveal how panic spreads through crowds.

\section{Emotional Component}

To represent the emotional component, the agent draws associations with certain behaviors and learns to act accordingly. The agent is conditioned in a certain way and develops instinctual feelings about particular events, people, or experiences. In evacuations, for example, people tend to be conditioned positively to associate authoritative figures with safety or even conditioned negatively to associate emergency evacuations with panic (37). Personal experiences dictate people's thinking processes and response to a given situation.

In the model, the agents tend to draw associations with the adverse behavior of agitation as their emotional component increases. Equation 2 represents this emotional component mathematically. [Equations 2 to 8 are formulas used in the model that were derived from the Agent_Zero methodology (9)].

emotional $=A+(\mathrm{LR}) * A *(\lambda-A)$

where

$$
\begin{aligned}
A= & \text { agent's emotional value } \\
\mathrm{LR}= & \text { learning rate, and } \\
\lambda= & \text { maximum associative strength attained through the learning } \\
& \text { process. }
\end{aligned}
$$

However, a gradual dissipation (extinction) of the adverse behavior begins whenever the conditioned associations subside. This dissipation occurs because of the presence of an authoritative figure or calming agents and results in a decline in the emotional affect. Therefore, Equation 3 incorporates an extinction rate, $\varepsilon$. In addition, the maximum associative strength, $\lambda$, becomes zero after all negative associations die out.

emotional $=A+(\mathrm{LR}) * A *\left(\varepsilon \cdot \mu \cdot N_{C}\right)(0-A)$

where

$\varepsilon=$ extinction rate,

$\mu=$ calming effect caused by the presence of calming agents, and

$N_{C}=$ number of calming agents within the agent's sightline (vision radius).
The vision radius restricts how far the agent can observe other agents in the model. The strength of the calming effect grows as the number of calming agents increases. In addition, with the presence of calming agents, the extinction rate increases and adverse behavior subsides more rapidly.

\section{Cognitive Component}

The cognitive component represents the reasoning of the agent based on an assessment of the behaviors of surrounding agents. While the emotional component focuses on the visceral feelings about what an individual already knows or has been conditioned to respond to over time, the cognitive component relies on observed evidence. In a pedestrian evacuation, as people navigate to an exit, the human tendency is to observe the behavior of others (38). An individual's actions are then based on this observed evidence.

To this end, the model uses local sampling to estimate mathematically the agitation levels exhibited by the surrounding agents. The agents assess the percentage of agitated actors within their vision radii. The model assigns a percentage to the observing agent based on the surrounding agents' adverse behavior. This value represents the agent's propensity to act in the same adverse manner. Equation 4 shows how the presence of calming agents decreases the influence of agitated evacuees:

cognition $=\left(\frac{N_{\mathrm{ag}}}{N_{\mathrm{tot}}}\right)^{\mu N_{C}}$

where

$$
\begin{aligned}
N_{\mathrm{ag}}= & \text { number of agents within the individual's vision radius with } \\
& \text { an agitation level above the threshold, } \\
N_{\mathrm{tot}}= & \text { total number of agents within the individual's vision radius, } \\
& \text { and } \\
\mu= & \text { calming effect }(\mu \leq 1) \text { of calming agents in the model. }
\end{aligned}
$$

The more calming agents there are, the less influence the agitated agents have. When no calming agents are within the individual's vision radius, the cognitive component simply becomes:

cognition $=\frac{N_{\mathrm{ag}}}{N_{\mathrm{tot}}}$

Using past experiences and the current observations of others' behavior in the immediate vicinity, an agent's characteristic attitude or temperament (disposition) is predicted by mathematically adding the emotional and cognitive outcomes of Equations 4 and 5 to obtain the agent's disposition, $D_{i}^{\text {solo }}$, as shown in Equation 6:

$D_{i}^{\text {solo }}(t)=$ emotional $_{i}+$ cognition $_{i}$

\section{Social Component}

Throughout the model, each eligible pedestrian agent has his or her own disposition. However, in evacuating crowds, individuals' actions are influenced to varying degrees by the actions of others (38). For the social component, the model takes into account not only the agent's own disposition but also the dispositions of nearby agents. In the model, the influence is spatial through physical proximity; that is, only neighboring agents affect each other. However, in an 
evacuation setting, influences can also be auditory (for example, a loudspeaker announcement), visual (such as exit signs), or tactile (nudging or pushing by others).

These influences are not considered in the current model but could easily be implemented by incorporating an additional influence (exogenous) on agent behavior, as in Equation 7. The model uses a weighted influence parameter to weight the dispositions of these nearby agents during the simulation run. An agent's total disposition, $D_{i}^{\text {total }}$, is defined in Equation 7.

$D_{i}^{\text {total }}(t)=$ emotional $_{i}+\operatorname{cognition}_{i}+\sum_{j \neq i} \omega_{j i}\left(\right.$ emotional $\left._{j}+\operatorname{cognition}_{j}\right)$

where $\left(\right.$ emotional $\left._{i}+\operatorname{cognition}_{i}\right)$ is agent $i$ 's disposition, and $\omega_{j i}$ and $\left(e^{2}\right.$ otional $\left.{ }_{j}+\operatorname{cognition}_{j}\right)$ are the weighted influence and dispositions of neighboring agents, respectively. This total disposition constitutes the social component, which rounds out the three necessary factors that will determine an agent's actions in the evacuation model.

\section{Action Rule}

Finally, because individuals have varying tolerance levels when faced with other people's behavior in a crowd, each agent has an action threshold. Compared with the agent's total disposition, the binary action rule determines whether the agent will act on a specific behavior. Equation 8 calculates the agent's net disposition, $D_{i}^{\text {net }}$, which includes the action rule used by each agent:

$$
D_{i}^{\mathrm{net}}(t)=\left(\begin{array}{c}
\text { emotional }_{i}+\operatorname{cognition}_{i} \\
+\sum_{j \neq i} \omega_{j i}\left(\text { emotional }_{j}+\text { cognition }_{j}\right)
\end{array}\right)-\tau_{i}
$$

where $\tau_{i}$ is the action threshold for agent $i$.

The agent's action threshold determines whether the agent will express adverse behavior in the model, in which case there will be some level of agitation and increase in speed toward the exit. Heterogeneous thresholds among individual agents introduce stochasticity into the model, as similar environmental and social conditions may induce agitation in certain agents while others with a higher threshold do not alter their emotional states.

\section{RESULTS}

The model presented in this study is an experimental implementation of Epstein's Agent_Zero framework to explore opportunities for understanding the spread of panic or other agitated behaviors throughout a pedestrian crowd. The results serve as a preliminary report on ongoing work in this area. Two measures constitute the primary focus of the initial model tests: evacuation time and density of pedestrians around the exit.

\section{Evacuation Time}

In the model, evacuation time is a measure of the number of simulated time-steps required to clear the modeled venue of all pedestrians (not including calming agents or peace officers). The parameters measured in the simulation account for $81 \%$ of the variation in evacuation time $\left(r^{2}=.81\right)$. Of the parameters, the weighted influence of the slowest member on overall group speed had no statistically significant effect. This finding is reflected in Table 1 with a $p$-value of .402 for the influence of the slowest member. As groups distribute more of the burden of slower members (for example, by carrying a small child), there is no noticeable effect on overall evacuation time.

The most influential factor, as shown in Table 1, is average group size. Group sizes are heterogeneous in the modeled environment. As the average size of groups of pedestrians increases, the evacuation time also increases. As groups get larger, the goals of agents to maintain group cohesion and accommodate the slowest member cause the group speeds to decrease. This decrease in overall speed likely accounts for the positive relationship between average group size and evacuation clearance times (Table 1).

The remaining factors-weighted influence, extinction rate, calming effect, number of calming agents, and vision radius - are all associated with the sociobehavioral aspects of the model. This model does not account for crushing behaviors and thus limits the interpretation of panic in this context. The negative relationship between evacuation time and weighted influence and vision radius suggests that the panic of neighbors affects an individual less during longer egress scenarios. These longer evacuations are also associated with higher extinction rates (dismissing panicked feelings after a certain amount of time) and higher calming effects by more calming agents.

Together, these sociobehavioral factors may appear counterintuitive. In the model, however, crowding at exits likely accounts

TABLE 1 Evacuation Time

\begin{tabular}{|c|c|c|c|c|c|}
\hline \multirow[b]{2}{*}{ Model } & \multicolumn{2}{|c|}{ Unstandardized Coefficients } & \multirow{2}{*}{$\begin{array}{l}\text { Standardized } \\
\text { Coefficients } \\
\beta\end{array}$} & \multirow[b]{2}{*}{$t$} & \multirow[b]{2}{*}{ Significance } \\
\hline & $\beta$ & Standard Error & & & \\
\hline Constant & 232.499 & 3.672 & & 63.310 & .000 \\
\hline Influence of slowest & 3.020 & 3.605 & .002 & .838 & .402 \\
\hline Weighted influence & -35.731 & 1.815 & -.058 & -19.690 & .000 \\
\hline Extinction rate & 10.828 & 1.815 & .018 & 5.967 & .000 \\
\hline Average group size & 58.376 & .192 & $\underline{.896}$ & 304.049 & .000 \\
\hline Calming effect & 21.113 & 1.815 & .034 & 11.635 & .000 \\
\hline Number of calming agents & .721 & .036 & .059 & 19.880 & .000 \\
\hline Vision radius & -1.253 & .454 & -.008 & -2.762 & .006 \\
\hline
\end{tabular}

${ }^{a} P$-values $<.05$ are significant. 
for these effects. Since these agents cannot harm one another to get out of the exits, longer evacuations are caused by longer lines. These are likely the result of large groups clogging egress routes. More calming agents cause the crowd to become calmer and be less influenced by agitated neighboring agents as they wait in long lines to leave. Future versions of this model will explore the possibility of aggression in the form of physical pushing to evaluate the effects of possible harm occurring in these contexts.

\section{Exit Density}

In the model, exit density is the number of pedestrians waiting in a region around the exit area to exit. The exit density measure is actually a count value for the number of time-steps that the exit region exceeded $75 \%$ occupied. This value was chosen to test the initial impact of Agent_Zero on crowding near the exit and will be calibrated to real-world scenarios in future versions of the model. The stochastic nature of exiting behaviors inspired by Agent_Zero made the exit density measure difficult to interpret. Using a multiple regression model, the $r^{2}$ value indicated that the modeled parameters only explained $56.2 \%$ of the variation in exit density. This likely comes from the interaction of Agent_Zero parameters that lead to highly stochastic outcomes in density around the exit.

Table 2 shows the output of a regression model that used all the available parameters in the simulation. The weighted influence of the slowest group member on the overall group speed (influence of slowest) does not significantly affect exit density. The two largest contributing factors are average group size and the number of calming agents (peace officers) in the model. As the average group size in the model increases (a heterogeneous factor with groups of differing sizes existing within the population), the density around the exit decreases. Since groups accommodate the speed of slower members as well as adjust speeds and trajectories to maintain group cohesion, larger groups generally move more slowly in the model. This behavior spaces out the agent groups as they approach the exit and leads to the exit area experiencing less time crowded with egressing pedestrians.

Alternatively, as the number of calming agents increases, the density around the exit increases. Since the calming agents migrate to the most agitated agents and navigate toward the exit as well, depending on an agents' threshold, the agitation levels and walking speeds of agents near a collection of calming agents will decrease. Over time as agents respond to the presence of these calming agents, the overall speed of the crowd will slow, causing the pedestrian agents to reach the exit simultaneously, thus producing higher densities around the exit. With the presence of calming agents walking toward the exit with the evacuees, the slower walking speeds allow for a more orderly and effective evacuation (39).

The less influential but still statistically significant factors in the model refer specifically to the sociobehavioral components based on the Agent_Zero framework. As agents place more emphasis on the agitation level of surrounding evacuees (weighted influence), exits have larger densities. Likewise, when agents retain the influence of neighbors' dispositions longer (lower extinction rates), densities increase. High exit densities are also associated with a large number of calming agents who have very little calming effect. The calming agents acting on the evacuees crowded around the exit decrease agitation, although there may be increased exit density.

Future versions of this model will focus more on the interaction between calming agents and egressing pedestrians to improve understanding of the consequences of these dynamics on evacuation clearance time and exit density. Furthermore, the calming agents themselves may become panicked and drastically affect the behavior of the egressing pedestrians. Future versions of the model should include this dynamic to understand its impact on clearance time and exit density.

\section{DISCUSSION AND CONCLUSIONS}

The preliminary results from the model indicate that average group size is the single most influential predictor of evacuation clearance time and exit density. These results follow from the fact that, in general, larger groups move more slowly and thus allow time for the exit to clear before reaching critical density, but also delay exit time. The finding of longer evacuation times for slower-moving crowds contradicts the established hypothesis that faster is slower (40) when it comes to pedestrian egress. According to the fasteris-slower hypothesis, as crowds move faster to exit, this behavior actually causes a longer exiting delay. Therefore, if the crowd slows down, there will be shorter delays causing a decrease in evacuation times, so that, essentially, slower is faster where slower speeds result in faster evacuation times.

The contradictory result of the model that slower is slower likely follows from two specific modeling features that require further investigation. First, the original model that developed the notion

TABLE 2 Exit Density

\begin{tabular}{|c|c|c|c|c|c|}
\hline \multirow[b]{2}{*}{ Model } & \multicolumn{2}{|c|}{ Unstandardized Coefficients } & \multirow{2}{*}{$\begin{array}{l}\begin{array}{l}\text { Standardized } \\
\text { Coefficients }\end{array} \\
\beta\end{array}$} & \multirow[b]{2}{*}{$t$} & \multirow[b]{2}{*}{ Significance $^{c}$} \\
\hline & $\beta$ & Standard Error & & & \\
\hline Constant & 124.766 & 1.942 & & 64.256 & .000 \\
\hline Influence of slowest & .428 & 1.906 & .001 & .225 & .822 \\
\hline Weighted influence & 22.766 & .959 & .106 & 23.728 & .000 \\
\hline Extinction rate & -14.687 & .959 & -.068 & -15.307 & .000 \\
\hline Average group size & -12.537 & .102 & -.553 & -123.504 & .000 \\
\hline Calming effect & -14.869 & .959 & -.069 & -15.497 & .000 \\
\hline Number of calming agents & 2.083 & .019 & .486 & 108.572 & .000 \\
\hline Vision radius & -1.127 & .240 & -.021 & -4.699 & .000 \\
\hline
\end{tabular}

${ }^{a} P$-values $<.05$ are significant. 
that faster is slower included friction variables to account for individual interactions among agents. This feature accumulated in dense situations, causing a phenomenon where individuals in the model struggled to exit. Second, early models did not account for group dynamics. The preliminary results of this study show that, even if the crowd moves slower to exit because of the nature of groups and reduced panic, the exit delay still remains and continues to negatively affect evacuation times. However, the slower-is-slower hypothesis suggests a more efficient evacuation with less congestion at the exits, encouraging a more orderly egress. These contradictory results may further emphasize the importance of including group variables as a consideration when studying egress scenarios. A group dynamicsinspired counterfactual to the faster-is-slower hypothesis deserves further attention and will be a focus of future iterations of the model presented here.

As a prototype, the current model has undergone limited formal validation and verification procedures. The most logical next step in this model development process is to calibrate the Agent_Zero parameters to reflect real-world scenarios. This is a difficult task because panic is not well documented in human crowds. The researchers will rely on available human research on crushing behaviors and panic in crowds, as well as the work of those studying relatable animal behaviors (41). Once these values have been calibrated in the model, it is hypothesized that Agent_Zero factors will have a more significant influence as predictors of evacuation clearance and exit density.

Qualitative approaches to validating the modeled behaviors are also part of the future development plans $(42,43)$. For example, utilization of survey-based qualitative data to capture aspects of decision making and human behavior that would be difficult to observe in video or laboratory settings is a viable approach for validation (43). Qualitative data relevant to the model presented here are currently being collected as part of the longer-term project. Other aspects of pedestrian crowd behavior in nonpanic scenarios can be helpful for validation purposes, such as group speeds, the effect of the slowest member on the group, and age-related factors. In addition, model performance comparisons can be applied for crowd behavior, such as well-used fundamental diagrams of observed speed-density-flow rate relationships. Lastly, the insight from emergency personnel subject matter experts through face validation could provide a level of confidence that the simulation model is performing credibly.

Preliminary validation of the pedestrian program used BehaviorSearch (44), based on the Active Nonlinear Tests (ANTs) approach to use search algorithms (genetic algorithms, simulated annealing, hill climbing, and random search) to test the extremes of the parameter values in the model (45). The ANTs approach revealed several sections of the vast parameter space - the many combinations of values in the model that contribute to the pedestrian egress and density outcomes-where unusual emergent behavior occurred in this model. In these cases, panic spread and dissipated rapidly, as if in waves, throughout the egressing agents. This type of emergent phenomenon is a common characteristic of agent-based models. It arises from the complexity of a multitude of interacting agent-level decisions contributing to unexpected global outcomes. The source of this particular emergent phenomenon in the pedestrian model will be a primary focus of future model development and research. At one extreme, it may reveal coding errors that have yet to be found; at the other, it may reveal interesting behaviors that arise when agents are acting independently on very simple egress rules that sow the seeds for pushing or crushing behaviors in crowds.

This model is part of a larger, ongoing project to understand pedestrian group egress. The findings related to group size and the influence of calming agents on exit density may have practical applications. More investigation into these phenomena is required; however, the results of this simulation may help venue managers and large event planners to use expected audience demographics related to group size to estimate more accurately the number of staff and peace officers needed at an event. This planning could support a more contextualized emergency evacuation and safe egress plan that adjusts dynamically based on the type of audience in attendance.

In the longer-term trajectory of this model, other advanced features that will be included are the potential for groups to split up and merge into other groups, reminiscent of early flocking models (46); adaptation of groups to be leaderless and instead congregate around shared goals of exiting and altruism; and further exploration of the implication of the Agent_Zero framework applied to larger, more complex pedestrian scenarios to anticipate the emergence of aggressive behaviors in agitated crowd egress scenarios. In addition, the current model uses agent speed as a proxy for agitated behaviors. Another variation of this behavior could build a feedback loop in which the speeds of agitated agents, in addition to their disposition, affect the panic levels of neighboring agents. Positive feedback loops such as these could have an escalating effect on panic and provide even more understanding about the sociobehavioral impacts of calming agents in pedestrian egress.

\section{ACKNOWLEDGMENT}

The authors thank the anonymous reviewers from the Standing Committee on Pedestrians for their constructive comments and helpful suggestions to enhance the paper.

\section{REFERENCES}

1. Vizzari, G., L. Manenti, K. Ohtsuka, and K. Shimura. An Agent-Based Approach to Pedestrian and Group Dynamics: Experimental and Real World Scenarios. Presented at 7th International Workshop on Agents in Traffic and Transportation, Elsevier, Ontario, Canada, 2012.

2. Helbing, D., and P. Molnar. Social Force Model for Pedestrian Dynamics. Physical Review E, Vol. 51, No. 5, 1995.

3. Hughes, R. L. The Flow of Large Crowds of Pedestrians. Mathematics and Computers in Simulation, Vol. 53, No. 4-6, 2000, pp. 367-370.

4. Hughes, R. L. A Continuum Theory for the Flow of Pedestrians. Transportation Research Part B: Methodological, Vol. 36, No. 6, 2002, pp. 507-535.

5. Vizzari, G., L. Manenti, and L. Crociani. Adaptive Pedestrian Behaviour for the Preservation of Group Cohesion. Complex Adaptive Systems Modeling, Vol. 1, No. 1, 2013, pp. 1-29.

6. Köster, G., M. Seitz, F. Treml, D. Hartmann, and W. Klein. On Modelling the Influence of Group Formations in a Crowd. Contemporary Social Science, Vol. 6, No. 3, 2011, pp. 397-414.

7. Seitz, M., G. Köster, and A. Pfaffinger. Pedestrian Group Behavior in a Cellular Automaton. In Pedestrian and Evacuation Dynamics 2012, Springer Science \& Business, 2014.

8. Reuter, V., B. S. Bergner, G. Köster, M. Seitz, F. Treml, and D. Hartmann. On Modeling Groups in Crowds: Empirical Evidence and Simulation Results Including Large Groups. In Pedestrian and Evacuation Dynamics 2012, Springer Science \& Business, 2014.

9. Epstein, J.M. Agent_Zero: Toward Neurocognitive Foundations for Generative Social Science. Princeton University Press, Princeton, N.J., 2013.

10. Matarić, M. J. Designing and Understanding Adaptive Group Behavior. Adaptive Behavior, Vol. 4, No. 1, 1995, pp. 51-80.

11. Thalmann, D.M.S.R. Crowd Simulation. Springer, London, 2013. http://dx.doi.org/10.1007/978-1-84628-825-8.

12. Curtis, S., S. J. Guy, B. Zafar, and D. Manocha. Virtual Tawaf: A Case Study in Simulating the Behavior of Dense, Heterogeneous Crowds. 
In Computer Vision Workshops (ICCV Workshops), 2011 IEEE International Conference, 2011, pp. 128-135.

13. Wijermans, N., R. Jorna, W. Jager, T. van Vliet, and O. Adang. CROSS: Modelling Crowd Behaviour with Social-Cognitive Agents. Journal of Artificial Societies and Social Simulation, Vol. 16, No. 4, 2013, p. 1.

14. Still, G. K. Crowd Dynamics. PhD dissertation. University of Warwick, United Kingdom, 2000.

15. Braun, A., S. R. Musse, L. P. L. de Oliveira, and B. E. Bodmann. Modeling Individual Behaviors in Crowd Simulation. In 16th International Conference on Computer Animation and Social Agents, IEEE, 2003, pp. $143-148$.

16. Raupp Musse, S., and D. Thalmann. Hierarchical Model for Real Time Simulation of Virtual Human Crowds. Visualization and Computer Graphics, IEEE Transactions, Vol. 7, No. 2, 2001, pp. 152-164.

17. Niederberger, C., and M. Gross. Hierarchical and Heterogeneous Reactive Agents for Real-Time Applications. Computer Graphics Forum, Vol. 22, No. 3, 2003, pp. 323-331.

18. Braun, A., B.E. Bodmann, and S. R. Musse. Simulating Virtual Crowds in Emergency Situations. In VRST '05 ACM Symposium on Virtual Reality Software and Technology, ACM, 2005, pp. 244-252.

19. Schweingruber, D. A Computer Simulation of a Sociological Experiment. Social Science Computer Review, Vol. 13, No. 3, 1995, pp. 351-359.

20. Villamil, M.B., S. R. Musse, and L. Luna de Oliveira. A Model for Generating and Animating Groups of Virtual Agents. In Intelligent Virtual Agents, No. 2792, Springer Berlin Heidelberg, 2003, pp. 164-169.

21. Elzie, T., E. Frydenlund, A. J. Collins, and R. M. Robinson. Conceptualizing Intra- and Inter-Group Dynamics Within a Controlled Crowd Evacuation. Journal of Emergency Management, Vol. 12, No. 6, 2014.

22. Collins, A., T. Elzie, E. Frydenlund, and R. M. Robinson. Do Groups Matter? An Agent-Based Modeling Approach to Pedestrian Egress. Transportation Research Procedia, Vol. 2, 2014, pp. 430-435.

23. Brogan, D., and J. Hodgins. Group Behaviors for Systems with Significant Dynamics. Autonomous Robots, Vol. 4, No. 1, 1997, pp. 137-153.

24. Hatfield, E., J. T. Cacioppo, and R. L. Rapson. Emotional Contagion. Studies in Emotion and Social Interaction Series, Cambridge University Press, New York, 1994.

25. Bratman, M.E. Intention, Plans and Practical Reason. Harvard University Press, Cambridge, Mass., 1987.

26. Shendarkar, A., and K. Vasudevan. Crowd Simulation for Emergency Response Using BDI Agent Based on Virtual Reality. Proc., Winter Simulation Conference, IEEE, Monterey, Calif., 2006.

27. Lee, S., and Y.-J. Son. An Integrated Human Decision Making Model for Evacuation Scenarios Under a BDI Framework. ACM Transactions on Modeling and Computer Simulation (TOMACS), Vol. 20, No. 4, 2010.

28. Okaya, M., and T. Takahashi. BDI Agent Model Based Evacuation Simulation. In 10th International Conference on Autonomous Agents and Multiagent Systems, No. 3, Richmond, SC, 2011, pp. 1297-1298.

29. Almeida, J.E., R. Rosseti, and A. L. Coelho. Crowd Simulation Modeling Applied to Emergency and Evacuation Simulations Using Multi-Agent Systems. Presented at 6th Doctoral Symposium on Informatics Engineering, Porto, Portugal, 2013.
30. Lee, R., and R. Hughes. Exploring Trampling and Crushing in a Crowd. Journal of Transportation Engineering, Vol. 131, No. 8, 2005, pp. $575-582$.

31. Lee, R. S. C., and R. L. Hughes. Prediction of Human Crowd Pressures. Accident Analysis \& Prevention, Vol. 38, No. 4, 2006, pp. 712-722.

32. Helbing, D., I. Farkas, and T. Vicsek. Simulating Dynamical Features of Escape Panic. Nature, Vol. 407, No. 6803, 2000, pp. 487-490.

33. Wilensky, U. NetLogo. Northwestern University, Evanston, Ill., 1999.

34. Cocking, C., and J. Drury. The Mass Psychology of Disasters and Emergency Evacuations: A Research Report and Implications for the Fire and Rescue Service. Fire Safety, Technology and Management, Vol. 10, 2008, pp. 13-19.

35. Smith, C.A., and P.C. Ellsworth. Pattern of Cognitive Appraisal in Emotion. Journal of Personality and Social Psychology, No. 48, 1985, pp. 813-838.

36. Tsai, J., N. Fridman, E. Bowring, M. Brown, S. Epstein, G. Kaminka, S. Marsella, A. Ogden, I. Rika, A. Sheel, M. Taylor, X. Wang, A. Zilka, and M. Tambe. ESCAPES-Evacuation Simulation with Children, Authorities, Parents, Emotions, and Social Comparison. In 10th International Conference on Autonomous Agents and Multiagent Systems, Taipei, Taiwan, 2011.

37. Klein, G. A. Sources of Power: How People Make Decisions. MIT Press, Cambridge, Mass., 1998.

38. Tsai, J., E. Bowring, S. Marsella, and M. Tambe. Modeling Emotional Contagion. In AAMAS 2011 Workshop on Multi-Agent Based Simulations (MABS), Taipei, Taiwan, 2011

39. Proulx, G., and J.D. Sime. To Prevent "Panic" in an Underground Emergency: Why Not Tell People the Truth? Fire Safety Science, No. 3, 1991 , pp. 843-852.

40. Helbing, D., I. Farkas, P. Molnar, and T. Vicsek. Simulation of Pedestrian Crowds in Normal and Evacuation Situations. Pedestrian and Evacuation Dynamics, Vol. 21, No. 2, 2002, pp. 21-58.

41. Shiwakoti, N., M. Sarvi, G. Rose, and M. Burd. Animal Dynamics Based Approach for Modeling Pedestrian Crowd Egress Under Panic Conditions. Transportation Research Part B: Methodological, Vol. 45, No. 9 2011, pp. 1433-1449.

42. Köster, G., F. Treml, M. Seitz, and W. Klein. Validation of Crowd Models Including Social Groups. In Pedestrian and Evacuation Dynamics 2012, Springer Science \& Business, 2014.

43. Frydenlund, E., T. Elzie, A. J. Collins, and R. M. Robinson. A Hybridized Approach to Validation: The Role of Sociological Research Methods in Pedestrian Modeling. Transportation Research Procedia, Vol. 2, 2014, pp. 697-705.

44. Stonedahl, F., and U. Wilensky. BehaviorSearch. Northwestern University, Evanston, Ill., 2013.

45. Lambrecht, M.R., P.L. Ivens, N. J. Vandaele, and J.H. Miller. Active Nonlinear Tests (ANTS) of Complex Simulation Models. Management Science, Vol. 44, No. 6, 1998, pp. 820-830.

46. Reynolds, C.W. Flocks, Herds and Schools: A Distributed Behavioral Model. In ACM SIGGRAPH 14th Annual Conference on Computer Graphics and Interactive Techniques, No. 21, ACM, 1987, pp. 25-34.

The Standing Committee on Pedestrians peer-reviewed this paper. 